\title{
SIMULATION ET DISSIMULATION DANS LES RÉCITS AUTOBIOGRAPHIQUES DES MEMBRES HONGROIS DE LA RÉSISTANCE FRANÇAISE ${ }^{1}$
}

\author{
Krisztián BENE \\ Université de Pécs, Pécs
}

\begin{abstract}
En): During the Word War II, the Internal and External Resistance have gathered the people who wanted to continue the fight against the Axis powers in France and on the other theaters of operations around the world. Beside of the French citizens a number of foreigners were in the ranks of these organizations. Among these there were a couple of thousand Hungarians who have fought for the liberty of France. The detailed analysis of these volunteers allows to better knowing their number and activity which were unknown until now. The discovery of the written works of these fighters made after the war contributes to the better knowledge of their history. At the same time the use of the simulation and the dissimulation as a result of the Hungarian authorities' pressure helps to understand the circumstances of the birth of these autobiographical works.
\end{abstract}

Keywords (En): World War II ; Resistance ; Free French Forces ; volunteers ; Hungarians

Mots-clés (Fr): Seconde Guerre mondiale ; Résistance ; Forces françaises libres ; volontaires ; Hongrois

\section{Introduction}

Malgré le fait que la France et la Hongrie sont des pays lointains qui ne maintiennent que des relations superficielles et parfois hostiles, les rapports historiques et culturels entre les deux pays existent déjà depuis un millénaire. D'une manière intéressante, ces rapports ne sont ni vraiment connus ni particulièrement intenses, ce qui peut certainement s'expliquer par plusieurs éléments séparant les deux États d'une manière ou d'une autre, et ces relations présentent un épisode pratiquement inconnu pendant la Seconde Guerre mondiale.

En France lors de la campagne de 1940, l'armée française subit une défaite écrasante en quelques semaines contre les troupes allemandes, par conséquent la France doit vivre sous l'occupation allemande, tandis qu'une partie minoritaire de sa population continue le combat contre l'Allemagne nazie. Auprès de ces derniers, on rencontre également un certain nombre d'étrangers vivant en France depuis un certain temps et qui ont l'intention de lutter pour la libération de leur nouvelle patrie soit en France soit à l'étranger.

$\mathrm{Au}$ sein de ces formations, on trouve également des Hongrois qui se trouvent en France et décident de participer au combat contre l'Allemagne. Étant donné que cette participation est très peu connue, nous commençons notre étude avec la

\footnotetext{
${ }^{1}$ La présente étude a été subventionnée par la Bourse de recherche János Bolyai de l'Académie hongroise des sciences.
} 
présentation de celle-ci pour que l'importance des ouvrages autobiographiques écrits par les participants soit plus évidente. L'ensemble de ces textes constitue un corpus singulier qui nous permet de découvrir les exemples particuliers de la simulation et de la dissimulation liés aux auteurs qui rédigent leurs œuvres dans des conditions spéciales.

\section{Les rapports militaires franco-hongrois pendant le conflit}

Lors de la Seconde Guerre mondiale, la France et la Hongrie se situent dans deux camps politiques opposés, car la Hongrie maintient un rapport étroit avec l'Allemagne nazie ce qui en fait un adversaire de la France pendant le conflit. Paradoxalement, les relations franco-hongroises sont correctes pendant les années de la guerre. Cette situation est liée au fait que la Hongrie ne déclare la guerre pour les puissances alliées qu'en 1941. Pour cette période, la France a déjà signé l'armistice avec l'Allemagne et l'Italie après une défaite écrasante subie en 1940. Ainsi, les deux États ont une relation neutre pendant l'époque étudiée (HOREL, $2013: 11)$.

La plupart des rapports militaires sont liés à la diaspora hongroise se trouvant en France pendant les années 1930-1940. Les membres de cette communauté arrivent en France en plusieurs vagues consécutives au cours de la première moitié $\mathrm{du} \mathrm{XX}^{\mathrm{e}}$ siècle en raison des circonstances économiques et politiques défavorables dans leur pays natal. Concernant leur nombre, il existe des estimations différentes. Selon le Consulat de Hongrie, il y a moins de quinze mille citoyens hongrois en France dans les années 1920. En même temps, selon les représentants de cette diaspora, le nombre des Hongrois atteint environ trente à cinquante mille personnes à la fin des années 1920 (JANICAUD, 2009 : 131-132) et quatre-vingts mille pendant les années 1930 (KOMJAT, PECSI, 1973: 17). La plupart de ces Hongrois travaillent dans l'industrie française dans la région de la capitale et au nord de la France (PECSI, 1980 : 249).

À partir de septembre 1939 jusqu'à mai 1940, environ deux mille Hongrois s'engagent dans l'armée française. Ces volontaires, comme les autres engagés étrangers, sont dirigés vers des unités spéciales récemment formées au sein de la Légion étrangère, les régiments de marche de volontaires étrangers (RMVE) (MAHUAULT, 2013 : 209-218). Après une instruction militaire hâtive et incomplète réalisée dans le Camp du Barcarès (en Pyrénées-Orientales), ces unités manquant de cadres professionnels et d'armes en quantité (MONTAGNON, 1999 : 211-215) sont dirigées au nord-est de la France. Avant leur dissolution du $1^{\text {er }}$ juillet 1940, les membres des trois régiments luttent avec acharnement contre les troupes allemandes, mais la plupart sont tués sur le champ de bataille ou faits prisonniers (COMOR, $2013:$ 752-753).

La France libre sous les ordres du général Charles de Gaulle est créée en été 1940 en Angleterre pour continuer le combat contre les membres de l'Axe aux côtés des forces armées britanniques. Dans les rangs de cette formation, on ne compte que sept mille soldats au début. Parmi ces volontaires, il y a des étrangers en nombre, y compris des Espagnols, des Polonais, des Belges, etc. (BROCHE, MURACCIOLE, 2010 : 554-555). Le nombre des Hongrois est relativement élevé au 
sein de cette unité particulière, selon des recherches récentes effectuées par l'auteur de cette étude, environ cent-cinquante personnes hongroises se trouvaient dans les rangs des Forces françaises libres. Cela signifie que les volontaires hongrois constituaient le $7^{\mathrm{e}}$ contingent le plus important parmi les engagés étrangers luttant dans les rangs de la France libre (voir SHD GR 16 P).

L'armée allemande occupe la France en été 1940, ce qui entraine la naissance d'une résistance clandestine regroupant ceux qui veulent lutter contre l'envahisseur allemand. Les Hongrois se trouvent parmi ces gens déterminés et établissent différents groupes de résistance surtout au sein du Mouvement des ouvriers immigrés (MOI) (SCHKOLNYK-GlangEAUd, 1990 : 30). Plus tard est créé le Mouvement pour l'indépendance hongrois (MIH) regroupant soixante-dix groupes résistants hongrois dont l'activité est constituée surtout d'actions clandestines pacifiques (GODO, 1980: 20-21). En même temps, les résistants hongrois participent également dans des actions armées contre les troupes allemandes. Ils contribuent à l'organisation des Milices patriotiques en 1944 pour prendre part à la libération du pays dans le cadre d'un soulèvement armé. Ces dernières sont particulièrement actives lors de la libération de Paris en août 1944 (FILYO, 1986 : 61-66). Après la libération de la capitale, le MIH crée une unité militaire régulière constituée de volontaires hongrois portant le nom de la compagnie Petőfi sous le commandement de László Marschall et d'Imre Palotás. Attachée au $3^{\mathrm{e}}$ bataillon du $51-22^{\mathrm{e}}$ régiment international de l'armée française, la troupe est utilisée à l'arrière-pays pour assurer la défense des lignes de communications et des bases militaires avant sa démobilisation en automne 1945 (SOTER, $1946: 199$ ).

\section{Les récits autobiographiques hongrois}

Les survivants de cette époque mouvementée ont le désir de partager leurs expériences avec le grand public, mais cette volonté est fortement influencée par deux circonstances: le nombre des survivants et la situation politique d'aprèsguerre. En ce qui concerne le premier, il est bien limité, car les combats étaient meurtriers pendant la guerre, surtout auprès des volontaires de 1939-1940, ainsi la plupart de ces combattants ne sont plus vivants au moment de la fin de la guerre. En même temps, l'évolution de la situation politique n'est pas favorable pour la publication de tels ouvrages, car la France et la Hongrie se trouvent dans deux camps politiques et idéologiques opposés, ainsi les autorités ne soutiennent pas l'édition des mémoires concernant les combats communs des deux pays. Par conséquent, la caractéristique la plus importante de ces œuvres est qu'elles sont très peu nombreuses, ainsi le corpus de notre étude est relativement petit (dans l'état actuel des recherches, une douzaine d'ouvrages).

Malgré le fait que leur nombre est restreint, ces livres offrent une grande variété quand on essaye de les catégoriser. Tous les ouvrages racontent la vie et l'activité des auteurs pendant la guerre, mais la transmission des informations est réalisée de plusieurs manières différentes. Dans la première catégorie, on trouve les mémoires dans lesquels les auteurs présentent les événements historiques où ils ont eu un certain rôle, mais l'accent est mis sur le contexte historique au lieu de 
leur vie personnelle. On peut y mentionner en premier lieu le livre de Yves de Daruvar, lieutenant du Régiment de Tirailleurs sénégalais du Tchad (DARUVAR, 1945).

Les autobiographies dans lesquelles les auteurs peuvent présenter aux lecteurs l'histoire, mais leur propre vie également sont également importantes. Ainsi, ce genre est plus personnel, mais contient souvent plus d'informations ailleurs inaccessibles, car fait référence à des lieux, des personnes et des événements réels : ils se différencient en cela des textes de fiction. L'autobiographie est menée à la première personne et suit en général un strict ordre chronologique. Ses exemples les plus illustres sont les œuvres de Lázár Endre Bajomi, ancien membre des RMVE et de la Résistance intérieure (voir par exemple BAJOMI, 1982 et BAJOMI, 1984), mais il faut également mentionner celle de Nicolas Dobo, ancien militaire mobilisé et résistant (DOBO, 1975) ainsi que Albert Haas, ancien résistant (HAAS, 1986).

Les romans historiques constituent la troisième catégorie. C'est une forme de romans qui prend pour arrière-plan un épisode de l'histoire pour lequel il ajoute des événements réels et fictifs. Normalement, l'auteur invente son double qui raconte sa vie de façon romanesque, ce qui lui permet la déformation de la vérité. Selon nos connaissances actuelles, ce sont les livres de Endre Murányi-Kovács (par exemple MuranyI Kovacs, 1957 et MuRANYI-Kovacs, 1958) et de Imre Bóc (BoC, 2012) qui racontent la vie des résistants avec les moyens de la fiction malgré le fait qu'ils partagent leurs propres expériences avec les lecteurs.

Le dernier groupe, celui des romans documentaires, contient le plus d'ouvrages qui essayent de donner un grand tableau historique basé sur la réalité, mais illustrés par des éléments fictifs, ainsi les recueils de mémoires sont présentés d'une façon romanesque. Les livres de Éva Bedecs (BEDECS, 1965), de János Békessy (alias Hans Habe, HABE, 1969) ou de József Árkus (ÁRKUS, 1964) sont les premiers à mentionner, mais d'une manière intéressante, certains des autres auteurs cités écrivent également des ouvrages de ce genre comme Bajomi ou Bóc. Pour la description de l'ensemble de ces catégories, on a choisi le terme du récit autobiographique qui résume bien les caractéristiques communes de ces ouvrages.

Étant donné la complexité de la situation politique après le conflit, il est difficile de publier les récits autobiographiques écrits par les anciens combattants hongrois sur leurs expériences vécues pendant la guerre. Bien que ces ouvrages soient destinés avant tout pour le public hongrois vivant en Hongrie, ils n'ont pas la possibilité de voir le jour dans le pays pendant longtemps à cause de l'évolution de la vie politique hongroise. Après la prise de pouvoir du parti communiste, les anciens résistants, malgré leur attachement à l'idéologie de gauche, sont traités comme des êtres suspects en raison de leur passé dans un pays capitaliste. Ainsi, la plupart de leurs textes restent pendant longtemps des manuscrits inédits en Hongrie, tandis que certains sont édités en France où ils ne rencontrent qu'un succès limité, car le public français ne s'intéresse pas à la description de l'activité des résistants hongrois. Cette position peu favorable par rapport à la publication des ouvrages mentionnés ci-dessus ne connaît un changement positif qu'à partir du milieu des années 1950, quand quelques romans historiques sont édités en Hongrie où leur réception est mitigée. Cette situation s'explique par plusieurs raisons. 
D'une part, ils sont lus par un public intéressé par ce genre, ainsi certains livres connaissent un tirage important. D'autre part, ces livres sont peu appréciés par la critique littéraire de l'époque parce qu'ils n'appartiennent pas au courant littéraire officiel du régime communiste. Par conséquent, ils sont considérés comme des ouvrages secondaires dont le contenu et le style sont bien inférieurs au niveau souhaité de la littérature communiste. En résultat de cette approche politique peu favorable, les œuvres de ces auteurs sont plus ou moins oubliées jusqu'à nos jours en Hongrie.

Bien qu'ils se trouvent dans une position marginalisée par la politique culturelle de l'époque, ces ouvrages illustrent bien l'identité des résistants hongrois. Malgré leur séjour relativement long, et souvent motivé par des raisons politiques, en France, ils gardent leur identité hongroise et rentrent en nombre en Hongrie au lendemain de la guerre. Apparemment, la vie culturelle intense au sein de la diaspora hongroise contribue à la sauvegarde de leur identité nationale. Il a fallu se contenter, pour l'analyse de l'utilisation des langues par ces volontaires hongrois, d'un corpus modeste constitué seulement de quelques livres écrits entièrement en français. Ce manque s'explique avant tout par le nombre réduit de ces personnes (une centaine au total). De plus, ces militaires ne forment pas d'unité autonome constituée exclusivement par des Hongrois, ainsi ils agissent dans un milieu entièrement francophone. Ce fait contribue certainement à la dégradation de leur connaissance de leur langue maternelle, ce qui est prouvé par la langue des rares publications réalisées par ces gens. Par conséquent, ces ouvrages (voir DARUVAR, 1945 ; MAGGIAR, 1947 ; MAGGIAR, 1984) sont écrits en français et sont destinés pour le public français.

La simulation et la dissimulation ont un rôle important dans ces ouvrages. Déjà l'utilisation de la langue est un signe précurseur de ce point de vue, car le choix de la langue de communication est un geste vers le public ciblé. Dans ce cas-là, on peut supposer que l'utilisation de la langue donnée est un moyen pour prouver à la société accueillante l'attachement et l'appartenance pour la communauté linguistique, culturelle et idéologique. Ainsi, tout au début, on rencontre deux groupes qui essayent d'obtenir une position plus favorable avec l'utilisation de la langue du pays qu'ils ont choisi comme domicile. D'une manière intéressante, les auteurs des ouvrages destinés au public francophone dissimulent souvent leur identité hongroise. Dans certains cas, ils ne précisent pas leur origine, comme par exemple Daruvár qui n'évoque que vaguement de cette question : «dans quelque nation d'Europe » (DARUVAR, $1945: 11$ ). On peut également mentionner Habe qui crée une identité totalement différente pour lui-même : «je suis venu de Suisse avec un passeport valide » (HABE, $1969: 17)$ «je vis depuis vingt ans en Suisse » (HABE, 1969 : 217). Bien que l'unité commandée par Raymond Maggiar dirige est surnommée le « Royal Hongrois » en raison des origines de son chef (BROCHE, MURACCIOLE, 2010 : 559-601), il ne fait aucune allusion à cette origine particulière dans ses livres consacrés à l'histoire de la formation (MAGGIAR, 1947 ; MagGiar, 1984). Cependant, les livres édités en Hongrie, en ciblant le public magyarophone accentuent toujours la présence des Hongrois dans la Résistance française, ainsi les lecteurs peuvent avoir une impression exagérée sur 
l'importance de la contribution hongroise aux efforts de guerre français, voir par exemple (MURANYI-KOVACS, 1958: 245-246) ou (BAJOMI, $1984: 104)$.

Étant donné que la plupart des Hongrois luttent dans la Résistance intérieure, leurs actions se déroulent dans la clandestinité absolue, car cette dernière est absolument nécessaire pour le succès de leur combat. Par conséquent, la dissimulation est le fil conducteur de la majorité de ces ouvrages, car les protagonistes sont obligés de cacher leur identité résistante devant les autorités vichystes et allemandes. Ainsi, les membres hongrois des groupes clandestins font de grands efforts pour se déguiser en citoyens français ordinaires qui n'ont aucune relation avec les résistants. Dans le cadre de cette action de dissimulation de grande envergure, ils utilisent souvent des pseudonymes (BoC, 2012 : 13-14), possèdent de fausses cartes d'identité souvent préparées par eux-mêmes (BAJOMI, 1982 : 460-461).

Le contexte politique a également une influence considérable sur les auteurs. Étant donné que la plupart des membres hongrois de la Résistance rentrent en Hongrie après la guerre, ils sont obligés de s'intégrer à la vie culturelle dirigée par le régime communiste. Par conséquent, leurs récits doivent être conformes aux attentes des dirigeants de la vie culturelle, sinon leurs textes ne seraient pas publiés. Les auteurs, pour pouvoir éditer leurs livres, doivent conclure un compromis : ils rédigent des récits contenant les tournures obligatoires du régime. Ainsi, les auteurs (ou les narrateurs) sont toujours des personnages ayant une conviction communiste (ou au moins socialiste) et le système occidental est présenté comme inférieur par rapport à l'idéal communiste (MURANYI KOVACS, 1957 : 296). L'autre élément commun est que, malgré l'attachement des auteursnarrateurs pour la France, les représentants officiels du pays et le général de Gaulle sont présentés d'une manière négative (ÁRKUS, 1964 : 26), (DOBO, 1975 : 29-30), (MURANYI-KovaCS, 1958 : 40-44). C'est-à-dire que les auteurs doivent payer un prix relativement élevé pour la publication de leurs textes : ils sont obligés de dissimuler leur éventuelle sympathie envers les autorités françaises et de simuler une conviction communiste.

Bien évidemment, on pourrait supposer que ces sentiments sont sincères, et dans certains cas éventuellement ils peuvent l'être, mais curieusement ces clichés ne sont pas présents dans les ouvrages parus en français. Dans ces derniers, c'est plutôt l'amour et le respect envers la France qui sont exprimés. Même si ces sentiments sont souvent sincères, on peut facilement admettre que l'accentuation de ces émotions est motivée par le désir d'intégration, ainsi elle appartient à la catégorie de la simulation. Par conséquent, nous pouvons constater sans exagération que l'utilisation de ces pratiques est avant tout le résultat de la pression politique. Pour conclure, on peut constater qu'en général c'est le désir d'intégration des auteurs qui les motive à utiliser ces moyens particuliers dans leurs œuvres.

\section{Conclusion}

Il faut avouer que les connaissances actuelles disponibles concernant le sujet des volontaires hongrois de la Résistance française pendant la Seconde Guerre 
mondiale sont loin d'être exhaustives, mais on peut noter que l'histoire de ces combattants particuliers constitue un univers varié, intéressant et peu connu.

Pour la découverte de ce milieu spécial, on a besoin des données conservées dans les archives, mais l'image ne peut pas être complète sans l'utilisation des ouvrages écrits par les participants. Bien que le nombre de ceux-ci soit limité, ils représentent d'excellentes sources d'informations qui peuvent élargir notre horizon de connaissance sur l'activité des résistants hongrois. Les récits autobiographiques créés par les combattants se divisent en plusieurs catégories qui sont pourvues des mêmes caractéristiques concernant l'utilisation des moyens de simulation et de dissimulation. On peut constater que l'emploi de ces deux pratiques n'est pas lié à la liberté artistique des auteurs, au contraire, il est le résultat du manque de liberté et se réalise surtout sous la pression des représentants de la vie politique hongroise.

Bien que cette influence politique nécessite une lecture attentive de la part des lecteurs pour pouvoir éviter les pièges idéologiques, ces ouvrages méritent l'attention, car ils peuvent considérablement enrichir nos connaissances sur un chapitre pratiquement oublié de l'histoire franco-hongroise.

\section{BIBLIOGRAPHIE}

ÁRKUS József (1964), A brüsszeli magyar század, Budapest, Zrínyi.

BAJOMI Lázár Endre (1982), Párizs csillagként reszkető, Budapest, Szépirodalmi.

BAJOMI Lázár Endre (1984), Tramontana. Magyar önkéntesek Franciaországban, Budapest, Zrínyi.

BeDECs Éva (1965), Magyarok Párizsért, Budapest, Zrínyi.

BoC Imre (2012), Grenoble, de l'occupation à la liberté, Grenoble, Presses universitaires de Grenoble.

BROCHE François ; MuRACCIOLE Jean-François (2010), Dictionnaire de la France libre, Paris, Robert Laffont.

COMOR André-Paul (éd.) (2013), La Légion étrangère. Histoire et dictionnaire, Paris, Robert Laffont.

DARUVAR Yves de (1945), De Londres à la Tunisie, carnet de route de la France Libre, Paris, Charles Lavauzelle.

DoBo Nicolas (1975), Orvos a fegyverek között, Budapest, Kozmosz.

FILYO Mihály (1986), Magyarok az európai antifasiszta ellenállási mozgalmakban, Budapest, Móra.

GoDo Âgnes 1980, Magyarok az európai népek antifasiszta harcában, Budapest, Zrínyi.

HaAs Albert (1986), Médecin en enfer, Paris, Presses de la Renaissance.

HABE Hans (1969), Ha elesnek mellöled ezeren..., Budapest, Kossuth.

Horel Catherine (2013), La France et la Hongrie : affinités passées et présentes, de saint Martin à Nicolas Sarkozy, Revue historique des Armées 270, Paris, Service historique la Défense, p. 5-13. 
JANICAUD Benjamin (2009), Les missions religieuses au sein de l'immigration hongroise en France (1927-1940), Cahiers de la Méditerranée 78, Nice, Centre de la Méditerranée Moderne et Contemporaine, p. 131-140.

Komjat Irén ; PECSI Anna (1973), A szabadság vándorai. Magyar antifasiszták Franciaországban 1934-1944, Budapest, Kossuth.

MAGGIAR Raymond (1947), Les fusiliers marins dans la division Leclerc, du débarquement en Normandie, en passant par Paris et Strasbourg jusqu'à Berchtesgaden, Paris : Albin Michel.

MAGGIAR Raymond (1984), Les Fusiliers marins de Leclerc : une route difficile vers de Gaulle, Paris, France-Empire.

MAHUAULT Jean-Paul (2013), Engagés volontaires pour la durée de la guerre à la Légion étrangère : (E.V.D.G.) 1780-71, 1914-18, 1939-45, Paris, Grancher.

MONCLAR Fabienne (2014), Monclar. Le Bayard du XX siècle, Versailles, Via Romana.

MONTAGNON Pierre (1999), La Légion étrangère de 1831 à nos jours, Paris, Pygmalion.

MuRANYI Kovacs Endre (1957), Szenvedélyes évek, Budapest, Szépirodalmi.

MuRANYI-KovaCs Endre (1958), Gilbert kapitány, Budapest, Móra Ferenc.

PECSI Anna (1980), Magyar ellenállók és partizánok Franciaország és Belgium antifasiszta küzdelmeiben, in : DOMBRADY Lóránd; NAGY Gábor (éd.), Fegyverrel a hazáért, Budapest, Kossuth-Zrínyi, p. 247-276.

SCHKOLNYK-GLANGEAUD Claude (1990), Les échanges culturels dans les milieux sympathisants communistes hongrois en France de 1936 à 1946, Cahiers d'études hongroises 2, Paris, Centre interuniversitaire d'Études hongroises, p. 27-33.

Service historique de la Défense. GR 16 P. Dossiers individuels du bureau Résistance.

SOTER István (1946), Magyar-francia kapcsolatok, Budapest, Teleki Intézet. 